
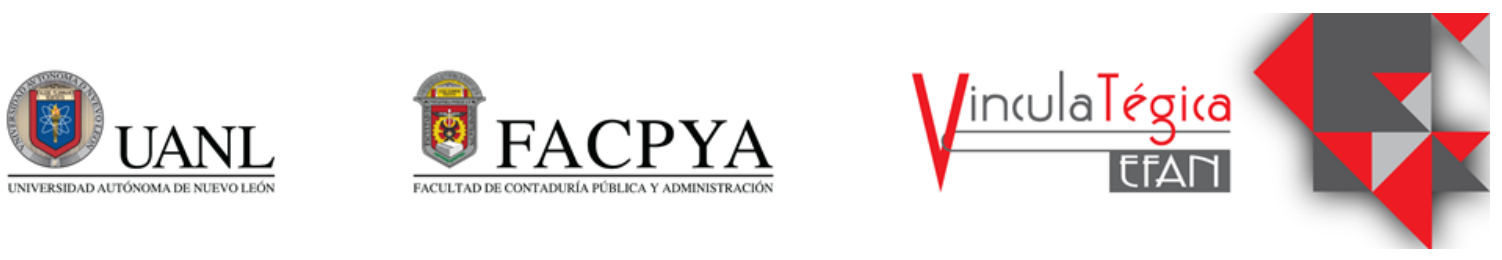

\title{
Diseño de instrumento de medición para analizar los factores que influyen en la productividad del sector automotriz de México
}

\author{
Loraine Gastell Piloto ${ }^{1}$, Lourdes Fabiola Espinoza Parada² y Jesús Gerardo Cruz-Álvarez ${ }^{3}$ \\ ${ }^{1}$ Universidad Autónoma de Nuevo León, Facultad de Contaduría Pública y Administración \\ Monterrey, Nuevo León, México, loraine.gastell@gmail.com, Av. Universidad S/N Col. Ciudad Universitaria, \\ (+52) 8111991535 \\ ${ }^{2}$ Universidad Autónoma de Nuevo León, Facultad de Contaduría Pública y Administración \\ Monterrey, Nuevo León, México, fabiola-espinoza@hotmail.com, Av. Universidad S/N Col. Ciudad \\ Universitaria, (+52) 6441715699 \\ ${ }^{3}$ Universidad Autónoma de Nuevo León, Facultad de Contaduría Pública y Administración \\ Monterrey, Nuevo León, México, jesusphd@ prodigy.net.mx, Av. Universidad S/N Col. Ciudad Universitaria,
} (+52) 8117786517

Información del artículo revisado por pares

Fecha de aceptación: junio-2021

Fecha de publicación en línea: diciembre-2021

DOI: https://doi.org/10.29105/vtga7.2-58

\section{Resumen}

En la actualidad las organizaciones se enfrentan con el reto de promover la mejora de la productividad entre los empleados. Por lo tanto, la productividad juega un papel crucial en la competitividad de la organización. La productividad de las industrias automotrices en México ha sido muy variable a lo largo de los años. Del año 2005 al año 2009, la misma comenzó a descender considerablemente. Probablemente las industrias del sector tomaron medidas, y en el período transcurrido desde el año al 2009 comenzó a incrementarse año tras año. No obstante, en el año 2016 la misma volvió a disminuir, lo cual evidencia una inestabilidad en la productividad de las empresas automotrices. El objetivo de esta investigación es diseñar un instrumento de medición para el análisis de los factores determinantes para la variación de la productividad en el sector automotriz de México.

Palabras clave: productividad, factores, industria automotriz, instrumento de medición.

\section{Abstract}

Organizations today are faced with the challenge of promoting improved productivity among employees. Therefore, productivity plays a crucial role in the competitiveness of the organization. The productivity of the automotive industries in Mexico has been highly variable over the years. From 2005 to 2009, it began to decline considerably. Probably the industries of the sector acted, and in the period elapsed from year to 2009 it began to increase year after year. However, in 2016 it decreased again, which shows an instability in the productivity of automotive companies. The objective of this research is to design a measurement instrument for the analysis of the determining factors for the variation of productivity in the automotive sector in Mexico.

Keywords: productivity, factors, automotive industry, measuring instrument.

JEL Codes: JEL: D2 - Production and organizations.

JEL: D24 - Production; capital productivity and total factor productivity; ability

M11 Production management 


\section{INTRODUCCIÓN}

La productividad de las empresas ha sido un foco de intensa investigación durante muchas décadas, no solo porque se identifica como una de las principales fuentes de competitividad, sino también por motivaciones empresariales, como orientar las inversiones hacia aquellos productos $\mathrm{y}$ servicios en los que se concentra la creación de valor, así como en la planificación de futuras operaciones industriales. Existe también un interés económico por la productividad que justifica su estudio y medición, ya que permiten hacer comparaciones y proponer políticas $\mathrm{o}$ programas públicos dirigidos a sectores industriales particulares.

Los fabricantes de automóviles globales han sido testigos de muchos cambios en los últimos diez años. La crisis financiera de los Estados Unidos (EE. UU.) en 2008 llevó al colapso de los tres grandes fabricantes de automóviles estadounidenses (GM, Ford y Chrysler). Toyota sufrió un declive en su estatus de marca global debido a la gran crisis de retiro del mercado de 2009-2010. Volkswagen, que había dado una buena batalla incluso durante la crisis de la deuda de la eurozona, se enfrentó a una situación difícil relacionada con el diesel en 2015.

Por el contrario, algunos fabricantes de automóviles como BMW, Hyundai y Kia han mostrado un crecimiento constante incluso frente a tales cambios ambientales externos. Por lo tanto, el éxito o el fracaso de los fabricantes de automóviles globales está determinado por cómo se enfrentan activamente al entorno rápidamente cambiante que rodea a la industria automotriz y maximizan la eficiencia operativa con recursos internos restringidos

En el año 2020 la producción total de vehículos y automóviles en todo el mundo fue más de 76 millones, lo cual constituyó una disminución de la producción de este sector, ya que en el 2019 se produjeron poco más de 91 millones, se registró una disminución del $13.65 \%$ en la producción automotriz a nivel mundial. Esta disminución puede deberse en gran medida a los cambios económicos sufridos a nivel mundial provocados por el impacto del COVID 19. La producción automotriz aumentó en un $10 \%$ en el período transcurrido desde el año 1999 al 2009, y en un 48\% del 2009 al 2019. En el caso de México la producción automotriz aumento en un $105 \%$ en el período de $1999-2020$, este aumento ha logrado colocar al país entre los primeros 5 países productores de vehículos a nivel mundial (OICA, 2021).

Por las razones antes mencionados este artículo está dirigido a estudiar e investigar realizando un instrumento de medición que ayude a investigar cuales son los factores que afectan la productividad del sector automotriz en México. Por lo que el objetivo de este sería diseñar un instrumento de medición que permita determinar los factores que influyen en la productividad de la industria automotriz en México. Para lograr el cumplimiento del objetivo se realizará una revisión de literatura donde se podrán determinar los factores que según diferentes autores influyen en el comportamiento de la productividad, posteriormente se diseñara el instrumento utilizando los criterios de los autores para redactar los ítems de esta.

\section{MARCO TEÓRICO}

\subsection{Variable dependiente: Productividad}

La definición de productividad se desarrolló en el siglo XVIII, derivado de la relación entre las entradas (recursos humanos y no humanos), los procesos de producción y las salidas (productos y servicios). El concepto de productividad se expandió con el tiempo a diferentes áreas. En macroeconomía la productividad consiste en la productividad parcial y total de los factores, y se ha utilizado para medir la competitividad de las empresas de ciertos países e industrias. La productividad parcial indica las salidas y las entradas individuales, especialmente la relación entre trabajo y capital, mientras que la productividad total es la relación existente entre múltiples entradas y salidas (Lee \& Leem, 2016).

La productividad es uno de los factores más importantes que afectan a cualquier empresa. Para mejorar la productividad, la 
producción en el sitio debe medirse regularmente y luego compararse con los puntos de referencia estándar aceptables. En su forma más simple, la productividad laboral podría definirse como las unidades de trabajo realizadas por las horas de trabajo divididas. Sin embargo, en realidad, la productividad laboral es un fenómeno mucho más complejo que depende en gran medida de factores muy diversos, como las condiciones del sitio, la competencia de los trabajadores, la disponibilidad de materiales, el clima, la motivación, la supervisión, por nombrar solo algunos. La gestión también afecta la productividad laboral (Enshassi et al., 2007). En el año 2018 se realizó un estudio donde se evalúa la contribución de la innovación en la productividad. Los resultados arrojaron que la productividad de la industria manufacturera de China se debe principalmente a las mejoras en la productividad dentro de la propia empresa. También se detectó que la mala asignación de recursos provoca grandes pérdidas en la productividad (Dai et al., 2018).

En la mayoría de los países la productividad laboral es de importancia crítica para la rentabilidad en la mayoría de los países. Muchos sectores de la industria han experimentado problemas crónicos, como una mala gestión, malas condiciones de trabajo inferiores y calidad insuficiente. Muchos investigadores, en la literatura, han identificado estos problemas como factores que afectan la productividad y también afectarán el desempeño de una empresa y la economía general del país. El desempeño de la mano de obra se ve afectado por muchos factores y generalmente está relacionado con el desempeño del tiempo, el costo y la calidad. Los factores de identificación y evaluación que afectan la productividad se han evidenciado en la última década; sin embargo, aún se necesita una comprensión más profunda para mejorar la productividad. Para lograr los ingresos esperados en cualquier empresa, es importante tener un buen control de los factores de productividad que contribuyen a la composición de la producción integrada, como mano de obra, equipo y flujo de caja (El-Gohary \& Aziz, 2013).

\subsection{Variable independiente: Recursos Humanos.}

En el campo de la economía laboral, se han realizado varios estudios, en muchos de estos se ha detectado una fuerte conexión entre los resultados de productividad de las empresas y los salarios promedios que se le ofrecen a la mano de obra que participa en las actividades laborales, plateando que las diferencias de salarios podrían estar estrechamente vinculadas a la variación de productividad (Card et al., 2018). También Bloom, Sadun, \& Reenen (2016) construyeron un índice de prácticas avanzadas que interpretaron como capital de gestión y comprueban que está estrechamente relacionado con las variaciones en la productividad de las empresas en varios países del mundo. Bloom et al. (2016) investigaron formalmente la medida en que la gestión, representada por un índice de adopción de prácticas de gestión avanzadas: influyen en la productividad de las empresas, a través de los canales de selección de la fuerza laboral y los pagos de salarios. Como resultados del estudio encontraron una fuerte relación entre la capacidad promedio de los empleados y las prácticas de gestión. En conjunto, sus resultados sugieren que el capital humano, especialmente el gerencial es importante en una empresa para la capacidad de mantener prácticas de gestión exitosas. Sin embargo, las mejoras en la productividad no se deben únicamente a la mano de obra utilizada por la empresa. En resumen, los trabajadores que poseen gran capacidad obtendrán mejores salarios y esto se deriva en un aumento de la productividad para la empresa.

La productividad de los trabajadores se ve afectada por la productividad de los compañeros de trabajo. Varios estudios que analizan un conjunto muy diverso de ocupaciones proporcionan evidencia de estos efectos secundarios de productividad. Los compañeros de trabajo son complementos o sustitutos en la función de producción en el lugar de trabajo, por lo que el esfuerzo de un trabajador afecta el resultado para otro trabajador (Amodio \& Martínez-Carrasco, 
2018). Por su parte (Arcidiacono, Kinsler, \& Price, 2017) utilizan a los equipos deportivos como un claro ejemplo de que los compañeros de trabajo si influyen en la productividad de todo un equipo. Menzel (2015) plantea en su investigación que los trabajadores pueden aprender unos de otros y, por lo tanto, ser más productivos cuando trabajan con compañeros altamente productivos. El comportamiento desempeña un papel importante, ya que los trabajadores pueden llegar a realizar menos esfuerzo si trabajan con compañeros que hacen un mayor esfuerzo. El esquema salarial, las políticas de despido o la gestión de recursos humanos en general influyen directamente en la productividad de un trabajador la cual deriva en la productividad de la empresa (Amodio \& Martinez-Carrasco, 2018).

\subsection{Variable independiente: Tecnología.}

Existe una relación estrecha entre el nivel de desarrollo económico y el nivel de desarrollo tecnológico. En este contexto, la tecnología se considera como la clave causal que permite la transformación eficiente de los insumos en productos, lo que la convierte en uno de los factores más importantes para mejorar la productividad, la calidad y la competitividad de las empresas. $\mathrm{La}$ experiencia exitosa de la transición del subdesarrollo al desarrollo de muchos países puede ser una valiosa lección para otros países que están persiguiendo una rápida evolución hacia la industrialización de sus economías y uno de los factores clave en esta rápida industrialización ha sido la adquisición masiva de tecnología. En este caso se revisó un estudio en el que se analiza en el que se explora la influencia de la adquisición de tecnología en el crecimiento en la productividad laboral de las empresas. Los resultados los resultados del estudio demuestran que la variable adquisición de tecnología tiene una influencia positiva $\mathrm{y}$ significativa en la productividad laboral de las empresas. Este hallazgo es importante, especialmente en términos de impulsar las economías de los países en desarrollo, ya que estos países tienden a tener pocas capacidades para la generación de su propia tecnología, dados sus bajos niveles de inversión interna en I + D. Por lo tanto, el desarrollo de políticas dirigidas a atraer tecnología y la compra de maquinaria y equipos que incorporan un alto conocimiento tecnológico puede contribuir al desarrollo económico de estos países (González et al., 2019).

Khandker \& Thakurata (2018) realizaron un estudio donde abordan el tema de la producción de arroz, que en este caso es el cultivo más importante de la India. Por lo que el aumento de la producción de arroz es muy importante para la seguridad alimentaria en la India. En la investigación los autores plantean que en este caso el aumento de la productividad de las empresas productoras de arroz a través de medios convencionales, como el aumento del área de cultivo, el riego y el uso de fertilizantes, enfrenta limitaciones, por lo que tratan de demostrar que la introducción de nuevas tecnologías aumenta la productividad de las empresas. Realizaron una experimentación y demostraron que utilizando nuevas tecnologías como la de producir arroz híbrido parecía tener buenos resultados, ya que tenía el potencial de aumentar la productividad en un $15-20 \%$ sin aumentar la superficie de cultivo.

\subsection{Variable independiente: Flexibilidad laboral-fabricación.}

Los sistemas de producción han evolucionado desde la producción artesanal hasta la producción adaptable en los últimos siglos. Hoy en día, la producción digital, la producción inteligente y la Industria $4.0 \mathrm{se}$ han convertido en un tema importante. Con el desarrollo de los sistemas de producción, la estrategia de producción pasó de la producción artesanal a la producción en masa y luego a través de la personalización en masa a la producción personalizada. Los mercados se convirtieron en mercados de compradores, lo que obligó a los fabricantes a la diferenciación de productos. Los fabricantes enfrentan el desafío de producir eficientemente diversas variantes de productos en varios tamaños de lotes. La Industria 4.0 ofrece una nueva forma de implementar una producción altamente 
flexible y eficiente. Para cumplir con el requisito de individualización, se debe implementar una producción orientada al cliente. Los sistemas de producción deben optimizarse para lograr una producción altamente flexible y eficiente. Esto lleva a la investigación de la flexibilidad y el diseño de sistemas de producción flexibles. En los últimos años, la personalización masiva ha sido considerada como uno de los principales impulsores de la próxima transformación de la economía mundial. Industria 4.0 se caracteriza por una fuerte individualización de los productos bajo la condición de una producción altamente flexible. El concepto de flexibilidad tiene efectos de gran alcance en el diseño de sistemas de producción en Industria 4.0. La industria 4.0 ofrece una nueva oportunidad para realizar producción flexible y eficiente. A través del modelado de los sistemas de producción en la Industria 4.0, los posibles retrasos, interrupciones e incumplimientos podrían pronosticarse $\mathrm{y}$ tratarse a tiempo. En consecuencia, se puede mejorar la productividad y la eficiencia de los sistemas de producción. La flexibilidad tiene un gran impacto en el diseño de sistemas de producción en la Industria 4.0 (Long et al., 2018).

Para hacer frente a las circunstancias económicas, sociales y tecnológicas altamente dinámicas de hoy en día, existe una creciente necesidad de que las empresas organicen de manera flexible sus demandas laborales. Las empresas pueden satisfacer ampliamente las demandas de flexibilidad laboral mediante estrategias y prácticas de flexibilidad laboral externas o internas. A través de la flexibilidad laboral externa, las organizaciones reaccionan a los cambios en la demanda de sus productos o servicios mediante el ajuste de la cantidad de trabajo que emplean haciendo uso de empleados temporales y estacionales, contratos a corto plazo, despidos, trabajo de agencia, trabajo independiente, subcontratación y tareas o trabajo. A través de las prácticas internas de flexibilidad laboral, las empresas se ajustan a los cambios en la demanda de producción mediante la reasignación, el cambio de personal y el rediseño de trabajos dentro de sus propias organizaciones. La productividad laboral se puede definir como el valor agregado por empleado de tiempo completo para las empresas, es una medida dinámica importante tanto del desempeño organizacional como de la competitividad. La productividad laboral a menudo se combina con el desempeño de innovación de la empresa porque se espera que las innovaciones exitosas de productos aumenten el valor agregado a la empresa. En este caso (Preenen, Vergeer, Kraan, \& Dhondt, 2017) investigaron la relación entre la flexibilidad laboral y la productividad. Los resultados del estudio mostraron una relación sólida y positiva entre las prácticas internas de flexibilidad y la productividad laboral, sugiriendo que las prácticas internas de flexibilidad laboral sí estimulan la productividad laboral.

\subsection{Variable independiente: \\ Proveedores.}

Los compradores pueden gestionar la calidad del producto que se obtiene de los proveedores de tres maneras: pueden mejorar la calidad de los proveedores directamente invirtiendo en los proveedores para mejorar un proceso/producto, pueden mejorar la calidad de entrada indirectamente al incentivar los esfuerzos de mejora de la calidad de los proveedores, y/o pueden controlar la calidad de los procesos subsiguientes inspeccionando las unidades entrantes. Los fabricantes se enfrentan a tareas importantes para gestionar la calidad de los proveedores, ya que la tercerización de la fabricación aumenta la dependencia de la calidad del producto de los proveedores. No obstante, la gestión de la calidad del proveedor se vuelve más difícil debido a la creciente escala de producción y la complejidad asumida por los proveedores. No es infrecuente que los incidentes de calidad del producto se relacionen con defectos de calidad del proveedor. La tendencia de la subcontratación aumenta la responsabilidad del proveedor sobre la calidad del producto y, por lo tanto, la importancia de la inversión directa de los compradores en el desarrollo del proveedor. La gestión de la calidad de los 
proveedores está estrechamente relacionada con los esfuerzos de inversión de los compradores para mejorar la calidad de los proveedores, junto con incentivos monetarios para motivar los propios esfuerzos de mejora de la calidad de los proveedores, así como con la inspección de la entrega de los proveedores para controlar la calidad saliente e influye directamente en la productividad de una industria (Lee, H. \& Li, 2018).

A pesar de los muchos beneficios de la subcontratación, las empresas siguen preocupadas por la falta de información crítica con respecto a los niveles de riesgo y las acciones de sus proveedores, que generalmente están a pocos enlaces de distancia. Por lo general, las empresas administran los riesgos de la cadena de suministro mediante el aplazamiento de los pagos a los proveedores hasta después de que se haya realizado la entrega. Aunque el enfoque de pago diferido desvía el riesgo del comprador al proveedor, las fallas recientes en la cadena de suministro sugieren que no necesariamente elimina el riesgo por completo. Por lo tanto, muchas compañías ofrecen incentivos y realizan inspecciones de las acciones tomadas en la fuente en lugar de esperar la entrega final. Además de los esfuerzos del proveedor, la calidad del producto final depende de varios otros factores que no son fácilmente visibles para el comprador. Estos pueden incluir la capacidad operativa, el nivel de experiencia en la planificación y la ejecución de la fabricación (Nikoofal \& Gümüş, 2018).

\subsection{Variable independiente: Innovación.}

La innovación es la búsqueda de la adopción y comercialización de nuevos procesos, productos y estructuras organizativas y conlleva incertidumbre. La innovación en sí misma y sus efectos en la productividad pueden ser heterogéneos entre las empresas. Existen cuatro tipos de innovación: producto, proceso, organización y marketing. Lejos de ser una preocupación solo de los países avanzados, los beneficios de los esfuerzos innovadores en términos de desempeño de las empresas adquieren importancia especialmente para las regiones en desarrollo del mundo, ya que la actividad de innovación es costosa para dichos países, debido a sus escasos recursos de tecnología y capital humano. Los esfuerzos de innovación pueden traducirse en ganancias de productividad para las empresas, de modo que las innovaciones pueden aumentar la eficiencia de las empresas y mejorar los productos que ofrecen, lo que aumenta la demanda y reduce los costos de producción (Fazlıoglu et al., 2018). La exposición a la crisis puede generar cambios significativos en el comportamiento innovador de las empresas, la capacidad de las empresas para transformar su actividad innovadora en ganancias de productividad también puede cambiar (Castellani et al., 2016).

Hay muchas maneras en que las empresas pueden aumentar su productividad y, por lo tanto, contribuir a la mejora de esta. Sin embargo, el motor de cambio más común e importante dentro de las empresas, particularmente en los países industrializados avanzados, es la introducción de nuevos productos, nuevos procesos o nuevas formas de hacer negocios: en otras palabras, innovación. El vínculo entre innovación y productividad generalmente se considera positivo y significativo. Los gobiernos independientemente del nivel de desarrollo del país están dispuestos a fomentar la innovación (Bartz-Zuccala et al., 2018). Las empresas mejor administradas son más propensas a innovar. Por lo tanto, un análisis de la productividad a nivel de empresa debería tener en cuenta la calidad de las prácticas de gestión de las empresas, además de la innovación. Mientras que los dos se correlacionan con un cierto grado, no son idénticos; cada empresa utiliza las prácticas de gestión, pero no todas las empresas innovan (Bloom et al., 2017). Las empresas de los países en desarrollo pueden imitar o adaptar tecnologías introducidas en otros lugares para ponerse al día con las empresas de los países avanzados, mientras que los últimos necesitan innovar en la frontera para avanzar más (Aghion, 2016) 


\section{MÉTODO}

En la revisión de literatura realizada en búsqueda de información sobre los instrumentos de medición, los autores abordaban las encuestas, estas son consideradas por ellos como medios que tienen como objetivo principal la recolección, el procesamiento y el análisis de la información. Esta información puede estar dada en unidades, en personas, en grupos de personas entre otros (Hernández et al., 2014).

El objetivo principal de una encuesta es investigar un segmento de cierta población, está población se supone que posee las mismas características que se desean estudiar en la población respectiva. En la investigación se detectó que existen varias etapas en la realización de la encuesta. La primera vendría siendo determinar el diseño y el alcance de esta. La segunda seria la elaboración del instrumento de medición que nos va a permitir llevar a cabo la recolección de la información (Crotte, 2011). En la tercera etapa de la realización de una encuesta se determina la forma en la que se aplicará el instrumento.

La encuesta es considerablemente empleada como medio de investigación, debido a que con la utilización de esta permite se pueden obtener datos de manera más veloz y eficiente. Este instrumento de medición tiene como ventajas que permite hacer aplicaciones colectivas y la recolección de datos acerca de muchas cuestiones a la vez (Anguita et al., 2003).

Una de las escalas de respuesta más populares utilizadas en el diseño de encuestas es la escala Likert. Rensis Likert fue un psicólogo social estadounidense, el cuál en la década de 1930, introdujo por primera vez una escala psicométrica de 5 puntos para medir una serie de proposiciones relacionadas con la actitud. La redacción utilizada en la escala inicial de Likert fue: aprobar fuertemente, aprobar, indeciso, desaprobar y desaprobar fuertemente. Con el tiempo, la redacción cambió de aprobar a acordar, lo que resultó en la escala Likert que se maneja en la actualidad: totalmente de acuerdo y de acuerdo, por un lado, y en desacuerdo y totalmente en desacuerdo por el otro lado, con un punto medio (Chyung et al., 2017).

Debido a su simplicidad y popularidad, la escala Likert tradicional se expandió en muchas variaciones de escalas tipo Likert. Estos se usan comúnmente en instrumentos de encuestas diseñados para: medir el desempeño de los empleados en el lugar de trabajo (Purdey, 2013), investigación de mercado (Garland, 1991), e investigación psicométrica (Kulas \& Stachowski, 2013).

\section{RESULTADOS}

Con el fin de demostrar el modelo que se propuso y evaluar las variables dependientes e independientes se desarrolló un instrumento de medición (ver Apéndice A). Para ellos la encuesta tiene un total de 48 ítems divididos en diez secciones, entre las que se encuentran las variables independientes y la dependiente, por otro lado, se encuentran las preguntas de carácter sociodemográfico de cada una de las unidades de medición de las empresas.

Además, la encuesta se elaboró para que se conteste a cada ítem empleando un grupo de respuestas dirigidas que fueron diseñadas basándose en la metodología de Likert. La escala de Likert utilizada es de 5 puntos que va desde "totalmente en desacuerdo" indicada con el número $1 \mathrm{y}$ termina con la descripción "totalmente de acuerdo", indicada con el número 5. Según Purdey (2013) las escalas de Likert de 5 y 7 puntos poseen puntuaciones medias iguales entre ellas. En este caso se decidió utilizar una escala de Likert de 5 puntos porque la mayoría de los estudios consultados habían utilizado este tipo de escala, además de que basándonos en la investigación de Purdey, daba lo mismo seleccionar cualquiera de las dos.

En la primera sección se incluye el nombre de la investigación y una descripción general de la encuesta, donde se explica la importancia que posee la misma. Por otro lado, se agradece de antemano a los colabores por su apoyo, se explica 
brevemente de qué manera serán procesadas las encuestas y con qué fines se utilizarán. Asimismo, explica la cantidad de reactivos o ítems que posee la encuesta y en cuantas secciones está dividida. La segunda sección aborda los datos de control por lo que la misma tiene preguntas de información genérica del encuestado y de la empresa, esta sección posee contiene 8 ítems. La tercera sección tiene como objetivo conocer aspectos generales de la productividad de la empresa, esta sección comienza con una breve descripción de productividad y algunos de los aspectos más destacados de sus definiciones. Esta sección contiene 7 ítems. Finalmente, las siguientes secciones están dirigidas a recolectar información específica de la investigación, está dirigida a medir factores determinantes de las variables independientes y dependiente. $\mathrm{La}$ variable recursos humanos posee nueve ítems y las tecnologías, flexibilidad laboralfabricación, proveedores e innovación poseen 6 ítems cada una.

\subsection{Operacionalización de las variables de la hipótesis.}

La operacionalización de un concepto se conoce como el proceso de evaluar conceptos y aplicarlo completamente. El proceso de operacionalización se lleva a cabo cuando se hace imprescindible determinar una variable observable que refleje al concepto, debido a que los conceptos no son abiertamente observables por ellos mismos (Mendoza \& Garza, 2009). En esta sección se evidencia la operacionalización de las variables basándose en toda la información presentada en los capítulos anteriores y utilizando toda la revisión de literatura realizada a lo largo de la investigación. El conjunto de preguntas que se utilizaron para medir las variables propuestas se obtuvo del marco teórico y de la opinión de expertos en el tema de investigación.

Además de las variables del modelo de investigación, se agregaron factores demográficos considerados como un factor importante que van desde los ítems 1 hasta el 8. Estos ítems se incluyeron teniendo en cuenta la opinión de los expertos que se consultaron para la validación del instrumento.

\subsection{Validez de contenido.}

En el presente, la validez de contenido es considerada por muchos como un requisito para desarrollar análisis de los resultados de las encuestas o instrumentos, aunque cabe destacar que no es el único recurso que se utiliza para esto. La validez de contenido no solo describe los ítems del instrumento de medición, la misma además incorpora indicaciones para la gestión de este y principios que sirven para que los mismos puedan ser corregidos y puntuados (Abad et al., 2011).

En esta investigación la validez de contenido que se aplicó fue la evaluación por expertos. El perfil de los expertos que revisaron todos profesionales con estudios de posgrados, algunos académicos e investigadores y otros con experiencia en el área de la industria automotriz. Para seleccionar a los expertos se utiliza el enfoque de (Mendoza \& Garza, 2009) en donde establece que los jueces pueden ser en primer lugar expertos académicos-prácticos de metodología de la investigación y/o expertos en el área en la que se esté realizando el estudio. Si el juez seleccionado fuera un experto académico-práctico el mismo debe tener nivel de postgrado o ser un investigador activo. En el caso de ser un experto empresarial en el tema que se está estudiando deben trabajar en un departamento o empresa relacionado con el área o sector de estudio y poseer una antigüedad mínima de 3 años. Además 'mínimo debe ser jefe de algún área de la empresa. Por otro es necesario que su nacionalidad sea mexicana y que la empresa donde labore sea igualmente mexicana.

Para el proceso de validación se contactaron 10 especialistas, de los cuales el $50 \%$ tiene estudios de doctorado y se encuentran enfocados en la investigación, por tanto, tienen dominio sobre los aspectos metodológicos. El 50\% son expertos del área automotriz que se encuentran trabajando en empresas pertenecientes a la 
industria automotriz. En el Apéndice B se describen, los nombres de los expertos, así como el área de especialidad y experiencia de cada uno de ellos. A los especialistas se les envío una encuesta de manera electrónica (ver Apéndice C). La encuesta estaba compuesta por ítems que se organizaron por variable, los cuáles poseían sus respectivas definiciones y estaban separados por secciones. Se evalúo el grado de relevancia de cada uno, utilizando una escala de 4 valores donde uno significa que es irrelevante; dos, poco relevante; tres, relevante y cuatro, muy relevante (Mendoza \& Garza, 2009).

Posteriormente cuando se recopilaron los resultados de todos los expertos se promediaron cada uno de los ítems y aquellos que obtuvieron valores por debajo de un valor de 3 se eliminaron del instrumento (Prat \& Doval, 2003). Con la validación de contenido se eliminaron 5 ítems $(12,13,42,44$ y 47) del instrumento porque tenían como resultado valores promedio menores a 3 . Por otro lado, los expertos hicieron recomendaciones para la redacción de algunos de los ítems y sugirieron agregar ciertos datos demográficos que en su opinión serian de gran ayuda para la investigación.

\section{CONCLUSIONES}

Hoy en día, el entorno económico y tecnológico que rodea a la industria automotriz está cambiando rápidamente. La mayoría de los fabricantes de automóviles se han concentrado en desarrollar automóviles electrónicos para superar el agotamiento del petróleo y abordar los problemas atmosféricos y ambientales en lugar de desarrollar automóviles con motores de combustión interna que utilizan gasolina y diesel como combustibles. Los sistemas de vehículos, como los automóviles inteligentes y los vehículos guiados autónomos, se están volviendo rápidamente inteligentes para satisfacer las crecientes expectativas de los consumidores. en cuanto a seguridad y conveniencia. Todo lo mencionado anteriormente se debe a que las empresas luchan por ser cada vez más competitiva. La competitividad está estrechamente relacionada a la productividad. En el caso específico de la industria automotriz en México son muy escasos los estudios de los factores que aumentan la productividad en la misma, en la revisión de literatura realizada en esta investigación se analizan algunos de ellos, pero sin embargo ninguno poseía un instrumento de medición diseñado, validado y confiable para determinar cuáles son realmente los factores que influyen en la productividad de la industria automotriz en México.

El instrumento de medición desarrollado en este estudio permitirá realizar un estudio de campo, para recabar datos de empresas del sector automotriz e inclusive de otros sectores. Las respuestas del estudio de campo se procesarían estadísticamente, y los resultados obtenidos se podrán utilizar para aprobar o disentir hipótesis y tomar decisiones. Este tipo de investigaciones permitirá a las empresas del sector en el que se aplique, tomar decisiones basadas en los factores de productividad que influyen. 


\section{REFERENCIAS}

Abad, F. J., Olea, J., Ponsoda, V., \& García, C. (2011). Measurement in Social and Educational Sciences.

Aghion, P. (2016). Competitiveness and growth policy design. Moving to the Innovation Frontier, 5.

Amodio, F., \& Martinez-Carrasco, M. A. (2018). Input Allocation, Workforce Management and Productivity Spillovers: Evidence from Personnel Data. The Review of Economic Studies, 85(4), 1937-1970.

Anguita, J. C., Labrador, J. R., \& Campos, J. D. (2003). La encuesta como tecnica de investigacion. Atención Primaria, 31 (8)(I), 527-538.

Arcidiacono, P., Kinsler, J., \& Price, J. (2017). Team, Productivity Spillovers in From, Production: Evidence Basketball, Professional. Journal of Labor Economics, 37(1), 191-225.

Bartz-Zuccala, P., Mohnen, H., \& Schweiger, W. (2018). The Role of Innovation and Management Practices in Determining Firm Productivity. Comparative Economic Studies.

Bloom, N., Brynjolfsson, E., Foster, L., Jarmin, R. S., Patnaik, M., Saporta-Eksten, I., \& Reenen, J. Van. (2017). What Drives Differences in Management? National Bureau of Economic Research, w23300.

Bloom, N., Sadun, R., \& Reenen, J. Van. (2016). Management as a Technology? National Bureau of Economic Research.

Castellani, D., Piva, M., Schubert, T., \& Vivarelli, M. (2016). R\&D and Productivity in the US and the EU: Sectoral Specificities and Differences in the Crisis. Papers in Innovation Studies, 15.

Card, D., Cardoso, A. R., Heining, J., \& Kline, P. (2018). Firms and labor market inequality: Evidence and some theory. Journal of Labor Economics, 36(S1), S13-S70.estimates. International Journal of Production Research, 40(12), 2705-2720.

Chyung, S. Y., Roberts, K., Swanson, I., \& Hankinson, A. (2017). EVIDENCE-BASED SURVEY DESIGN: THE USE OF A MIDPOINT ON THE LIKERT SCALE. Performance Improvement, 56(10), 15-23. https://doi.org/10.1002/pfi

Crotte, I. R. R. (2011). Elementos Para El Diseño De Técnicas De Investigación: Una Propuesta De Definiciones Y Procedimientos En La Investigación Científica. Tiempo de Educar, 12(24), $277-$ 297.

Dai, X., Sun, Z., \& Liu, Ha. (2018). Decomposing the contribution of firm innovation to aggregate productivity growth: the case of Chinese manufacturing industry. Applied Economics Letters, 26(7), 543-548.

Fazlıoğlu, B., Dalgıç, B., \& Yereli, A. (2018). The effect of innovation on productivity: evidence from Turkish manufacturing firms. Industry and Innovation, 26(4), 439-460.

González, J., Vila, M., \& Guisado, M. (2019). Exploring the complementarity between foreign technology, embedded technology and increase of productive capacity. Technological and Economic Development of Economy, 25(1), 39-58.

Hernández, S. R., Baptista, L. P., \& Fernández, C. C. (2014). Metodología de la Investigación. McGraw-Hill Interamericana, 533.

Khandker, V., \& Thakurata, I. (2018). Factors encouraging complete adoption of agricultural technologies: The case of hybrid rice cultivation in India. Journal of Agribusiness in Developing and Emerging Economies, 8(2), 270-287.

Lee, C., \& Leem, C. (2016). An empirical analysis of issues and trends in manufacturing productivity through a 30-year literature review. South African Journal of Industrial Engineering, 27(2), 147-159.

Lee, H., \& Li, C. (2018). Supplier quality management: Investment, inspection, and incentives. Production and Operations Management, 27(2), 304-322.

Long, F., Zeiler, P., \& Bertsche, B. (2018). Realistic modelling of flexibility and dependence in production systems in Industry 4.0 for analysing their productivity and availability. Proceedings of the Institution of Mechanical Engineers, Part O: Journal of Risk and Reliability, 232(2), 174- 
184.

Mendoza, J., \& Garza, J. B. (2009). La medicion en el proceso de investigacion cientifica: Evaluacion de validez de contenido y confiabilidad. Innovaciones de Negocios, 6(1), 17-32.

Menzel, A. (2015). Organizational Learning: Experimental Evidence from Bangladeshi Garment Factories.

Nikoofal, M., \& Gümüş, M. (2018). Quality at the source or at the end? Managing supplier quality under information asymmetry. Manufacturing \& Service Operations Management, 20(3), 498516.

OICA. (2021). International Organization of Motor Vehicle Manufacturers.

Prat, R. S., \& Doval, E. D. (2003). Construcción y análisis de escalas. Analisis Multivariable Para Las Ciencias Sociales, 43-89.

Preenen, P., Vergeer, R., Kraan, K., \& Dhondt, S. (2017). Labour productivity and innovation performance: The importance of internal labour flexibility practices. Economic and Industrial Democracy, 38(2), 271-293.

Purdey, B. (2013). Occupant stimulus response workplace productivity and the vexed question of measurement. Facilities, 31(11-12), 505-520. https://doi.org/10.1108/F-03-2012-0021 


\section{APÉNDICE A}

Figura 1. Instrumento de medición

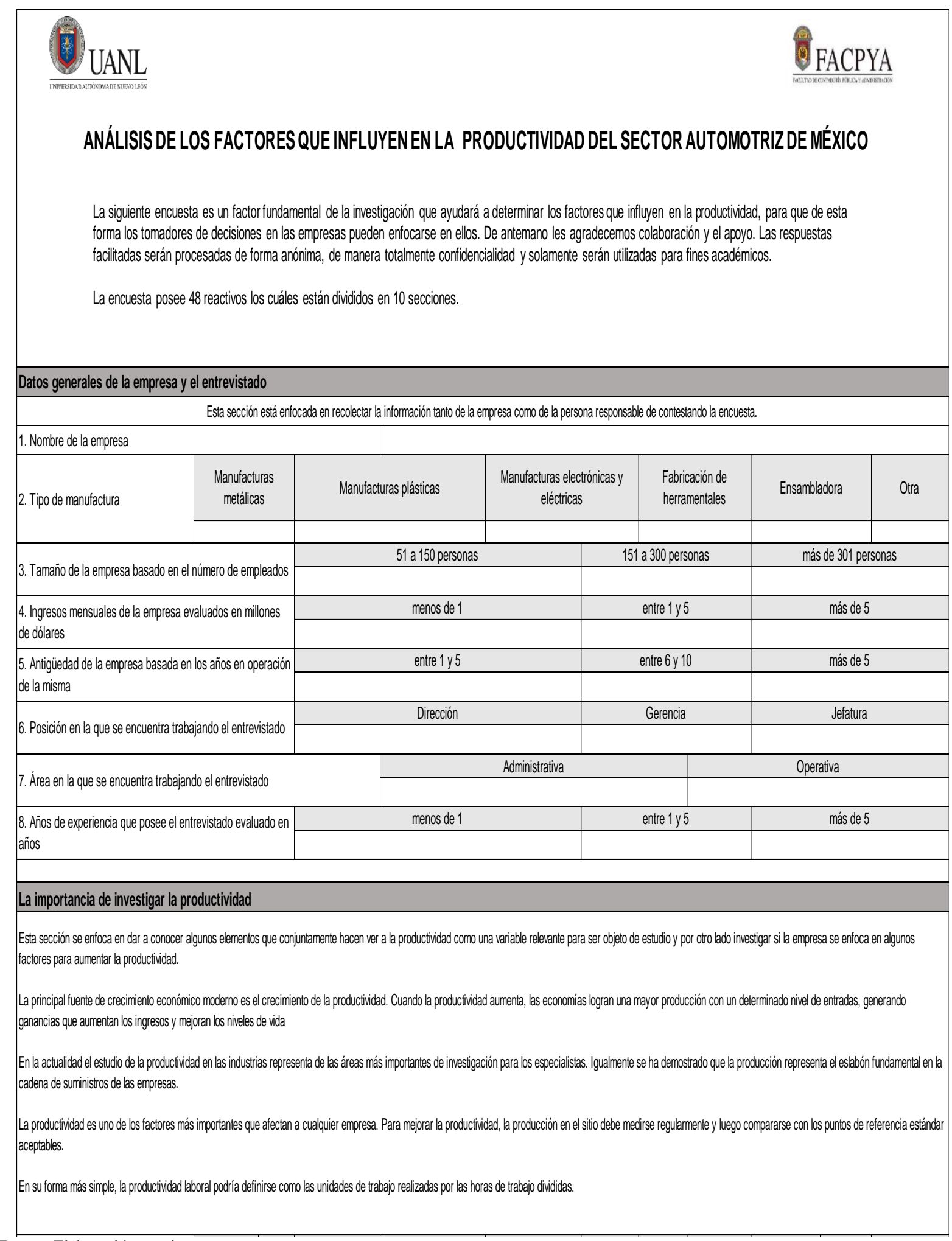

Fuente: Elaboración propia. 
Figura 2. Instrumento de medición

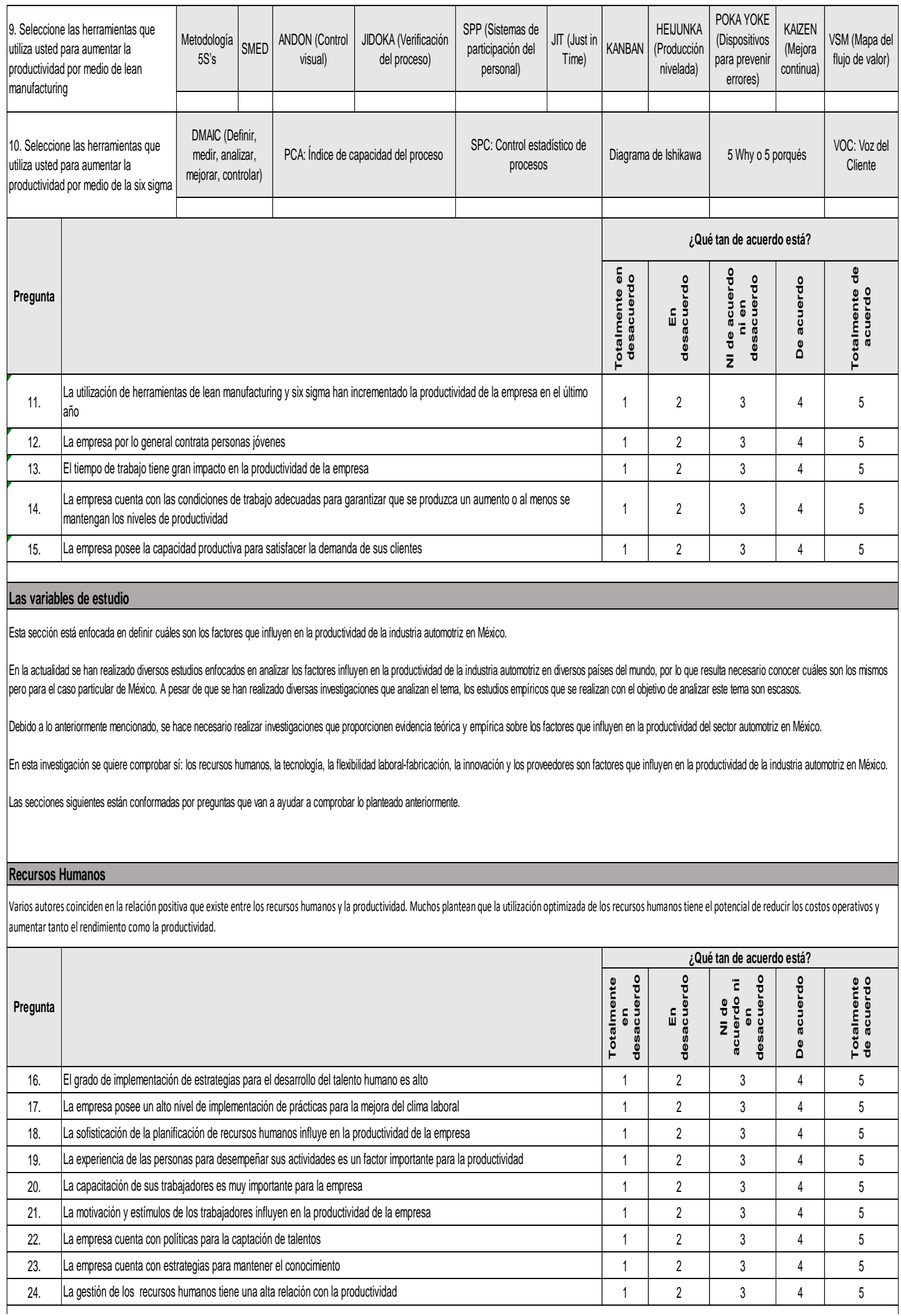

Fuente: Elaboración propia. 
Figura 3. Instrumento de medición

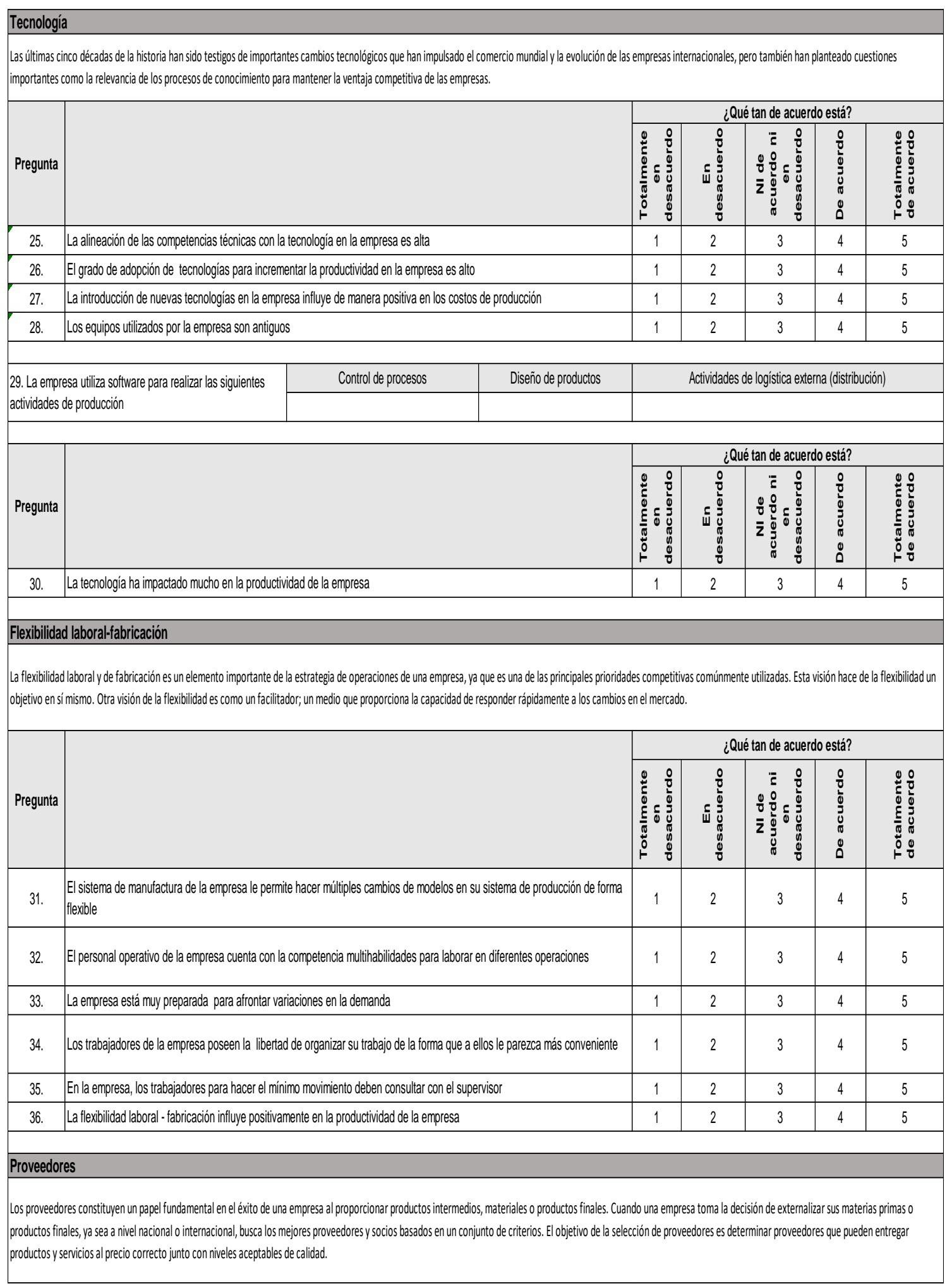

Fuente: Elaboración propia. 
Figura 4. Instrumento de medición

\begin{tabular}{|c|c|c|c|c|c|c|}
\hline \multirow[b]{2}{*}{ Pregunta } & & \multicolumn{5}{|c|}{ ¿Qué tan de acuerdo está? } \\
\hline & & $\begin{array}{ll}0 & 0 \\
5 & 0 \\
0 & 0 \\
0 & 0 \\
\varepsilon & 0 \\
0 & 0 \\
0 & 0 \\
0 & 0 \\
0 & 0 \\
1 & 0\end{array}$ & 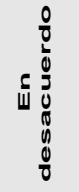 & 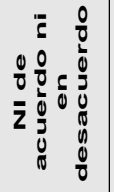 & $\begin{array}{l}0 \\
0 \\
\frac{0}{0} \\
0 \\
0 \\
0 \\
0 \\
0\end{array}$ & $\begin{array}{ll}0 & 0 \\
0 & 0 \\
0 & 0 \\
0 & 0 \\
5 & 0 \\
0 & 0 \\
0 & 0 \\
0 & 0 \\
1 & 0\end{array}$ \\
\hline 37. & El nivel de evaluación y desarrollo de proveedores es alto & 1 & 2 & 3 & 4 & 5 \\
\hline 38. & El proceso de evaluación y desarrollo de proveedores, les ha ayudado a mejorar los rechazos & 1 & 2 & 3 & 4 & 5 \\
\hline 39. & Los proveedores que posee la empresa tienen conocimiento de los índices de productividad que maneja la misma & 1 & 2 & 3 & 4 & 5 \\
\hline 40. & $\begin{array}{l}\text { La utilización en la producción de materia prima que no cumpla con los estándares de calidad influye en la productividad de la } \\
\text { empresa }\end{array}$ & 1 & 2 & 3 & 4 & 5 \\
\hline 41. & $\begin{array}{l}\text { Los incumplimientos de horarios de entrega de materia prima pactados con los proveedores atectan la productividad de la } \\
\text { empresa }\end{array}$ & 1 & 2 & 3 & 4 & 5 \\
\hline 42. & El proceso de evaluación y desarrollo de proveedores influye mucho en la productividad & 1 & 2 & 3 & 4 & 5 \\
\hline \multicolumn{7}{|c|}{ Innovación } \\
\hline \multicolumn{7}{|c|}{$\begin{array}{l}\text { La innovación es critica para el desarroll o económico. El crecimiento de la productividad a través de la eficiencia, las ganancias, la creación y satisfacción de nuevos deseos, ylas innovaciones que impulsan estos cambios son cruciale. } \\
\text { para la competitividad de la empresa y el crecimiento económico a largo plazo. }\end{array}$} \\
\hline \multirow[b]{2}{*}{ Pregunta } & & \multicolumn{5}{|c|}{ ¿Qué tan de acuerdo está? } \\
\hline & & 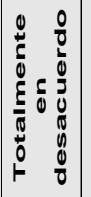 & 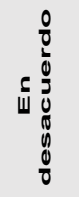 & 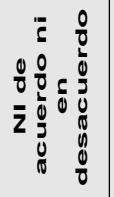 & $\begin{array}{l}0 \\
0 \\
\frac{0}{0} \\
0 \\
0 \\
0 \\
0 \\
0\end{array}$ & 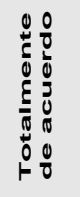 \\
\hline 43. & La empresa tiene implementado un proceso para canalizar las ideas, que logre captar e instrumentar ideas de mejora & 1 & 2 & 3 & 4 & 5 \\
\hline 44. & El ritmo de implantación de las ideas es muy alto & 1 & 2 & 3 & 4 & 5 \\
\hline 45. & Las herramientas y los sistemas que sustentan los procesos de innovación de la empresa son suficientes & 1 & 2 & 3 & 4 & 5 \\
\hline 46. & La empresa reconoce y estimula a los trabajadores que contribuyen al proceso innovador dentro de la misma & 1 & 2 & 3 & 4 & 5 \\
\hline 47. & $\begin{array}{l}\text { El horario de trabajo de la empresa permite que los trabajadores puedan tener oportunidad de idear soluciones creativas y } \\
\text { nuevas a problemas que puedan estarse presentando en la empresa }\end{array}$ & 1 & 2 & 3 & 4 & 5 \\
\hline 48. & La innovación tiene mucha relación con la productividad & 1 & 2 & 3 & 4 & 5 \\
\hline \multicolumn{7}{|l|}{ Fin } \\
\hline \multicolumn{7}{|c|}{$\begin{array}{l}\text { Se le agradece de antemano haberle dedicado una parte de su valioso tiempo a responder esta encuesta. Indudablemente su ayuda es de vital importancia para el desarrollo de la investigación. } \\
\text { Su respuesta nos ayuda a entender el comportamiento de los factores que afectan la productividad para de esta forma garantizar que los tomadores de decisiones de las empresas del sector automotriz en México van a tener a su } \\
\text { disposición información relevante acerca de los factores que afectan a la productividad. }\end{array}$} \\
\hline
\end{tabular}

Fuente: Elaboración propia 